\title{
Endoscopic Balloon Dilation for Crohn's Disease-Associated Strictures
}

\author{
Thomas Klag, Jan Wehkamp and Martin Goetz \\ Department of Internal Medicine I (Gastroenterology, Hepatology, Infectious Diseases), University Hospital Tübingen, Tübingen, Germany
}

Management of intestinal strictures associated with Crohn's disease (CD) is clinically challenging despite advanced medical therapy directed toward mucosal healing to positively influence the natural course of CD-associated complications. Although medical therapy is available for inflammatory strictures, therapy of fibrostenotic strictures is the domain of surgery and endoscopy. Endoscopic balloon dilation (EBD) has been recognized as a well-established first-line procedure in terms of safety and efficacy. Although surgery is a valuable treatment modality for the management of $\mathrm{CD}$-related strictures, $\mathrm{EBD}$ can help prevent multiple surgical interventions, which might in the long-term lead to a risk of short bowel syndrome. In this review we discuss requirements, techniques, safety, shortand long-term outcomes, as well as combinations of this procedure with surgical and medical treatment in CD-associated intestinal strictures. Clin Endosc 2017;50:429-436

Key Words: Crohn’s disease; Stricture; Stenosis; Balloon dilation; Constriction, pathologic

\section{INTRODUCTION}

Crohn's disease (CD), a chronic inflammatory condition of the intestines, is characterized by an overarching transmural inflammation of intestinal tissue, which can result in inflammatory or fibrotic stenosis of the small and large bowel, leading to clinical symptoms like postprandial abdominal pain, as well as ileus. ${ }^{1}$ Approximately $30 \%$ of patients diagnosed with $\mathrm{CD}$ are observed to develop intestinal strictures within 10 years of disease onset. ${ }^{2}$ Anti-inflammatory therapy using anti-tumor necrosis factor (TNF)-alpha antibodies aimed at mucosal healing has improved medical treatment options for inflammatory stenosis in patients presenting with $\mathrm{CD}$; however, controversies abound in literature regarding the increased likelihood of stenosis or strictures associated with its use. ${ }^{3}$ Therefore, a significant proportion of patients require surgical

Received: September 11, 2017 Accepted: September 17, 2017

Correspondence: Martin Goetz

Department of Internal Medicine I, University Hospital Tübingen, OtfriedMüller-Straße 10, Tübingen 72076, Germany

Tel: +49-7071-2980584, Fax: +49-7071-2925034

E-mail: martin.goetz@med.uni-tuebingen.de

(c) This is an Open Access article distributed under the terms of the Creative Commons Attribution Non-Commercial License (http://creativecommons.org/ licenses/by-nc/3.0) which permits unrestricted non-commercial use, distribution, and reproduction in any medium, provided the original work is properly cited. treatment for severe stenosis (resection or stricturoplasty), resulting in loss of extensive functional segments of the intestine potentially leading to short bowel syndrome. ${ }^{3}$ Notably, postoperatively, symptomatic recurrence of stenosis is a common clinical difficulty observed in approximately $40 \%$ of patients within 4 years after surgery. ${ }^{4}$ In these cases recurrent surgery might be needed, although it is associated with the risk of short bowel syndrome.

Endoscopic balloon dilation (EBD) has been developed as a widely used alternative in the treatment of strictures in patients with $\mathrm{CD}$ with overall favorable results in terms of safety, efficacy, and patient satisfaction. ${ }^{5.7}$ However, several reports in literature describe the need for surgery after balloon dilation in approximately $25 \%$ of patients at the time of 3-4 year follow-up. ${ }^{8}$ Therefore, the time and clinical situation/setting that is suitable to perform a balloon dilation are subjects of controversy among $\mathrm{CD}$ experts. The advantage of an $\mathrm{EBD}$ is that it is a safe and bowel-conserving procedure, particularly useful in the treatment of short stenosis. ${ }^{9}$ However, disadvantages of an EBD include the probable need for repeated dilations, as well as the need for surgery following dilation in a significant number of patients. ${ }^{10}$ These controversial points of view have led to differing/variable indications for an EBD in clinical practice as shown by Bettenworth et al., indicating that surgeons, when compared to gastroenterologists, favor surgery 
for de novo strictures. ${ }^{11}$

This review will present an overview of our experiences and current literature regarding indications and dilation techniques, safety of the procedure, short- and long-term results of an $\mathrm{EBD}$ in patients diagnosed with $\mathrm{CD}$, the pros and cons of balloon dilation vs. surgery in patients diagnosed with $\mathrm{CD}$, as well as features of medical interventions in the management of CD-related strictures.

\section{SETTING AND TECHNIQUES OF ENDOSCOPIC BALLOON DILATION IN PATIENTS WITH CROHN'S DISEASE}

Appropriate patient selection is an important and one of the most challenging factors that determines the success of an EBD. A fundamental prerequisite is that patients should be clinically fit to undergo this procedure in that if EBD-associated complications were to arise, the patient should be fit to undergo surgery. Ideally, EBD should not be performed in an emergency setting. ${ }^{12}$ An ideal candidate is a patient with a long history of $\mathrm{CD}$ ( $>15$ years) who is likely to develop a fibrostenosis as opposed to an inflammatory stenosis, one with a short stenosis $(<4 \mathrm{~cm})$ with minimal inflammation and no history of surgery, or a patient in whom a clinical complication is attributable to a single anastomotic stricture. ${ }^{12}$ In patients with strictures amenable to EBD, in addition to a short length $(<4 \mathrm{~cm})$ with minimal inflammation, malignancy or high-grade dysplasia must be ruled out, and the strictures should be straight, in line with the bowel lumen, without a fistula orifice close to it (distance from fistula if present should be $>5 \mathrm{~cm}$ ) and without an abscess in the stricture area. ${ }^{12,13}$

Prior to performing an EBD, routine laboratory testing for total blood counts, metabolic profile, and coagulation profile is necessary to avoid the risk of bleeding evaluate the risk of conscious sedation. A detailed history of the patient's individual disease course is also required, especially with respect to prior surgeries indicating altered bowel anatomy. Institution of antibiotic prophylaxis prior to EBD is only recommended in patients with a high risk of endocarditis, and other such conditions.

Abdominal imaging should be performed prior to an EBD. In Europe, ultrasonography is commonly performed to screen for abscesses or other complications of $\mathrm{CD}$. When performed by experts, ultrasonography is comparable to magnetic resonance imaging (MRI) to rule out abscesses in the stricture area, which would be a potential contraindication for an EBD..$^{14}$ Furthermore, contrast-enhanced ultrasonography can successfully distinguish between fibrosing and inflammatory stenosis. ${ }^{15}$ These methods can help to characterize lesions prior to performing an EBD to estimate the inflammatory activity. Although computed tomography is the most common imaging method used in the USA to rule out an abscess and to locate the number and length (extent) of the stenosis, this modality cannot differentiate between inflammatory and fibrotic stenosis in most cases. ${ }^{12}$

In some cases, patients with $\mathrm{CD}$ are known to suffer from concomitant diseases that might require the use of drugs such as anticoagulants. Effective management of these agents is a common clinical difficulty prior to performing an EBD. For example, recommendations of the American Society of Gastrointestinal Endoscopy suggest that concomitant use of aspirin is not associated with a higher risk of bleeding complications. However, in patients using thienopyridines or warfarin, bridging therapy, e.g., with aspirin and heparin may be recommended after weighing the risk-benefit ratio of bleeding vs. thromboembolic complications in the individual patient. ${ }^{16}$ The same principle is applied to the use of novel oral anticoagulants. ${ }^{17}$ However, anticoagulation should be restarted as soon as the risk of bleeding is low again (normally within
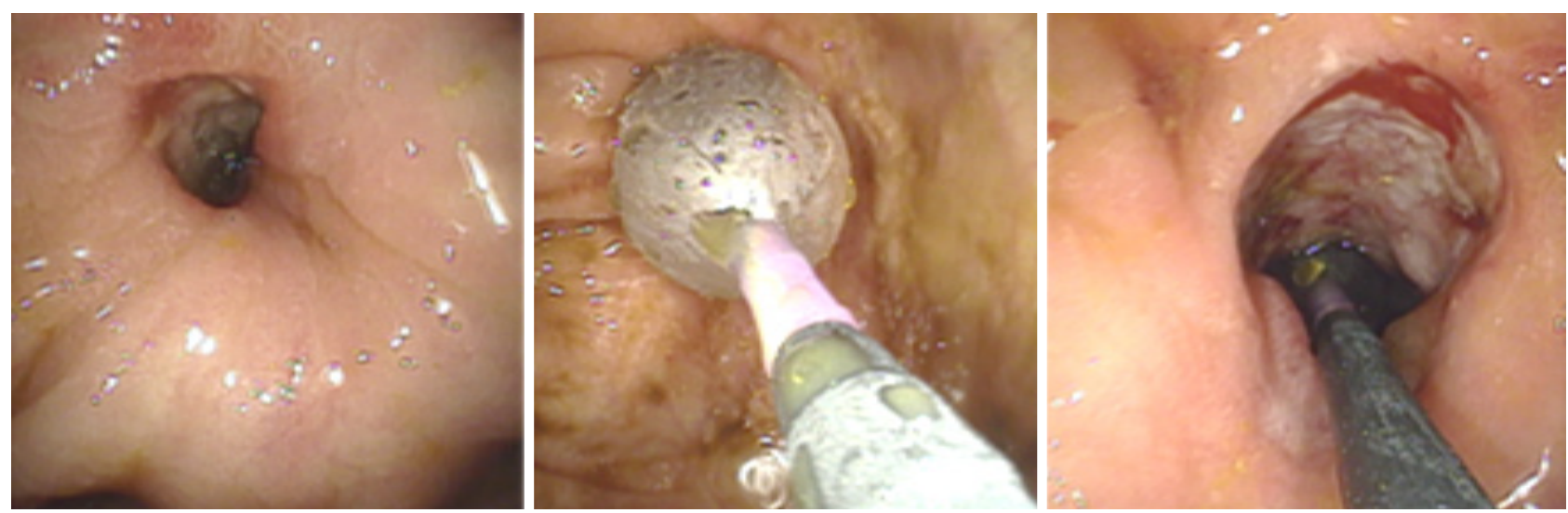

Fig. 1. Antegrade balloon dilation (from left to right). A balloon is gently introduced into the stricture followed by hydrostatic dilation. 
24-48 hours after intervention).

Although two balloon systems are available (over-the-wire balloon catheter and through-the-scope balloon [TTS]), the latter is more commonly used because of the ease of practical application and safety. ${ }^{6.12}$ The TTS system allows direct endoscopic visualization during the dilatation procedure. In principle, retrograde and antegrade balloon insertion into the stenotic area are the methods commonly employed. The antegrade approach does not allow the endoscope to pass across the stenosis. In this case a balloon is gently introduced into the stricture followed by hydrostatic dilatation (Fig. 1). Importantly, the balloon should not be forced across the area of stenosis/ stricture to avoid perforation and via falsa formation. This is particularly important in patients presenting with angulated and extended strictures. Wire-guided balloon introduction is helpful in complex strictures or when the proximal aspect of the stricture cannot be clearly visualized through the stenosis.

The retrograde dilatation approach can be used when the endoscope can pass across the stenosis-the endoscope is passed across the stenosis followed by withdrawal of the endoscope while positioning the balloon within the stricture.

For each approach, the size of the balloon, time of dilation, and graded vs. non-graded dilation is individually tailored by the endoscopist in routine clinical practice, based on the stricture size, length, and anatomy. ${ }^{6,11,12}$ However, a balloon size of $18-20 \mathrm{~mm}$ for the colon and $12-15 \mathrm{~mm}$ for small bowel strictures are the most commonly used sizes, based on reports of different studies and meta-analyses.- ${ }^{5-711}$ Larger balloons, for example, those that measure $25 \mathrm{~mm}$ are associated with a higher complication rate (bowel perforation, bleeding), ${ }^{18}$ and do not correlate with better long-term success. ${ }^{12}$ Therefore, a maximal balloon size of $12-20 \mathrm{~mm}$ is considered appropriate for management of most strictures. To date, there is no consensus regarding the time of dilation and a graded (beginning with a small balloon with an incremental increase in balloon size) vs. non-graded approach. Most authors report use of a graded approach, as shown in systematic reviews and expert surveys. ${ }^{7,11}$ Duration of dilatation varies between 20 seconds to 3 minutes. Most commonly, approximately 1 minute of dilation is used, ${ }^{5,11}$ and only a few endoscopists favor a longer dilation time.?

We usually use carbon dioxide $\left(\mathrm{CO}_{2}\right)$ insufflation to perform an EBD. This is beneficial in terms of reduced abdominal discomfort during and after endoscopic treatment because $\mathrm{CO}_{2}$ is more rapidly absorbed compared to ambient air, particularly during treatment of small bowel strictures that are treated using balloon-assisted enteroscopy. ${ }^{6,19}$ Fluoroscopy is not mandatory, but may be helpful in cases of altered anatomy and for delineation of difficult strictures using a contrast catheter (Fig. 2). Radiation exposure should be maintained at a minimal level.

\section{SAFETY OF ENDOSCOPIC BALLOON DILATION IN PATIENTS WITH CROHN'S DISEASE}

EBD is a minimally invasive procedure; however, bowel perforation and/or severe bleeding have been reported as typical complications associated with dilatation. Several me-
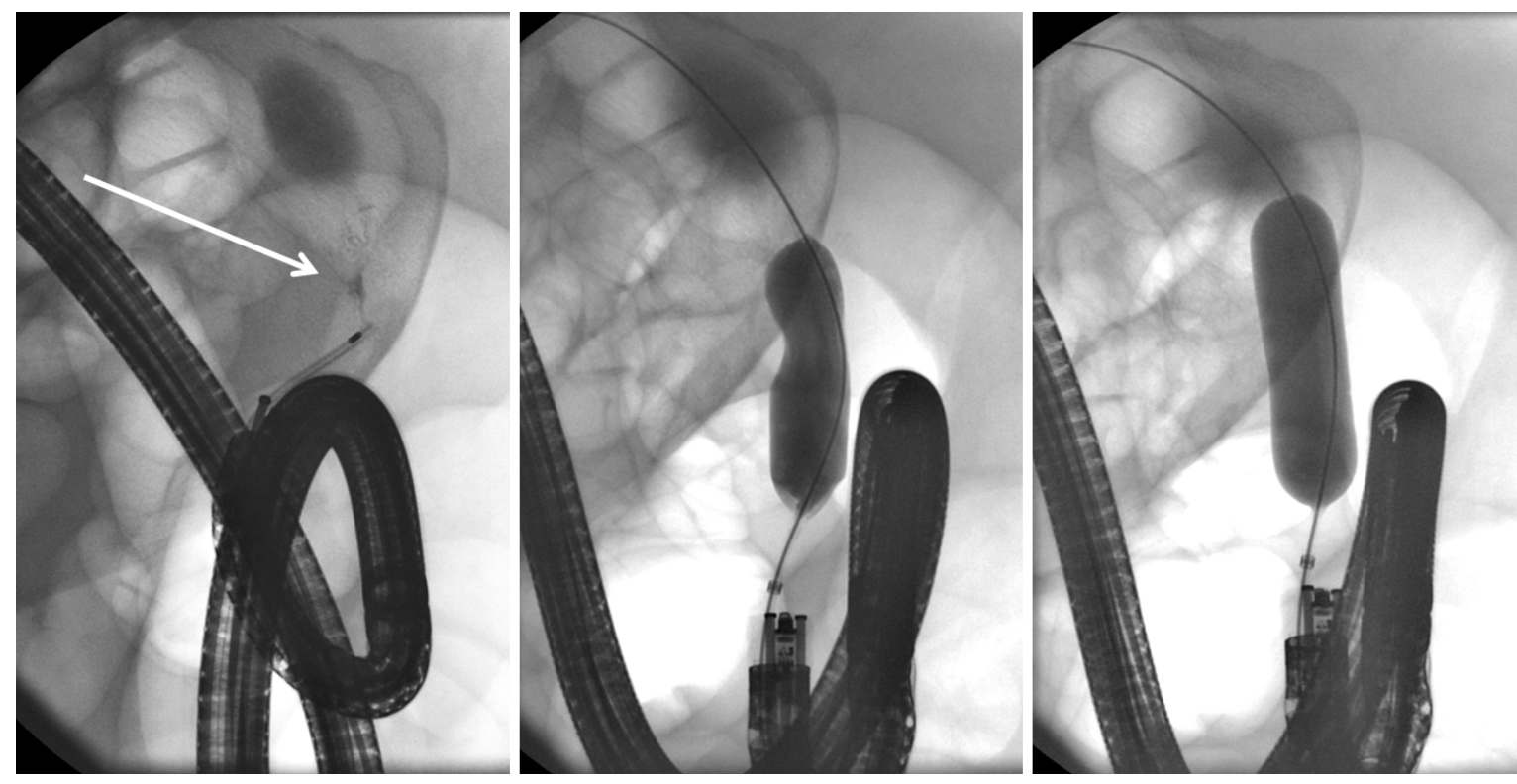

Fig. 2. A segment of short stenosis is delineated using injection of contrast via a catheter (arrow). Note the endoscope is in a torqued position secondary to postoperative adhesions. A balloon is advanced over a wire and carefully inflated until the indentation subsides. 
Table 1. Safety Requirements in Order to Avoid Complications of Endoscopic Balloon Dilation in Crohn's Disease

\begin{tabular}{|c|c|}
\hline 1 & The endoscopist and the team should be well trained \\
\hline 2 & Plan endoscopic balloon dilation in an elective setting, if possible \\
\hline 3 & Consider discontinuation or switch of anticoagulation \\
\hline 4 & Screen for cardio-vascular or pulmonary high risk patients regarding sedation \\
\hline 5 & Thrive for excellent bowel preparation \\
\hline 6 & Persist on imaging and previous patient data regarding abscess, fistula, inflammatory aspect of stricture and bowel anatomy \\
\hline 7 & Use carbon dioxide (or insufflate as little air as possible) \\
\hline 8 & Do not force the balloon across a stricture in a blind fashion—use wire guided, contrast assisted or retrograde dilatation instead \\
\hline 9 & Do not dilate too aggressively (Do not "over-dilate", rather schedule two sessions for step-wise dilatation) \\
\hline 10 & If complications are suspected aim for immediate diagnosis and treatment (e.g., clip application etc.) \\
\hline 11 & $\begin{array}{l}\text { If complications are suspected, patients should stay on liquid diet for about } 48 \text { hours after intervention (second look endoscopy or } \\
\text { surgery may be required) }\end{array}$ \\
\hline
\end{tabular}

Own experiences and partly Adapted from Chen et al. ${ }^{12}$

ta-analysis and reviews have shown a good safety profile of EBD. In their analysis of 24 studies involving 1,163 patients, Navaneethan et al. showed that major adverse events (perforation, hemorrhage necessitating intervention or blood transfusion, abscess, fistula, and/or sepsis) occurred in only $4 \%$ of patients. ${ }^{7}$ Similarly, Morar et al. in their meta-analysis investigating 25 studies with 2,664 dilations performed in 1,089 patients, reported a complication rate of approximately $4 \%{ }^{20}$ Our own experience has shown similar rates of complications. Retrospective analysis of our cohort between 2005 and 2013 with 118 dilations performed in 46 patients revealed a patient-related complication rate of $4.6 \% .{ }^{5}$ Both complications were perforations; however, the perforation in one patient could be managed conservatively. To date, there is no consensus regarding the management of patients after an EBD to screen for potential complications (e.g., using computed tomography, clinical observation, and/or evaluation of blood counts among other tests). Our own approach involves observing patients overnight in the hospital with clinical monitoring only, without performing routine laboratory testing or imaging studies. However, based on our experience and that of other researchers, ${ }^{12}$ complications can be avoided through adoption of several practices to minimize their occurrence, as outlined in the discussion above and summarized in Table 1.

\section{SHORT-TERM RESULTS OF ENDOSCOPIC BALLOON DILATION IN PATIENTS WITH CROHN'S DISEASE}

Although the definition of short-term efficacy of EBD has not been standardized, it can be defined as the technical success of the procedure itself. Most reports define short-term efficacy as the passage of the endoscope across the site/area of stenosis/stricture after dilatation. ${ }^{6}$ Relief of clinical symptoms (e.g., abdominal pain, nausea, vomiting, and/or bloating) after dilatation could be used as markers of the success of an EBD to define short-term efficacy of the procedure. ${ }^{6}$ Based on these heterogeneous definitions across studies, a few meta-analyses estimate a short-term success rate of $90 \%$ in terms of a technical response and a rate of approximately $70 \%$ in terms of a symptomatic response..$^{20}$ Navaneethan et al. in their systematic review reported a primary technical success rate of $89 \%$ associated with an EBD. ${ }^{7}$ However, symptomatic response rates were observed to vary between $42 \%-90 \%{ }^{6}$ Our own data confirm/validate these results. The primary success rate for 118 dilations performed for 69 strictures showed a primary technical success rate of $89.6 \%{ }^{5}$

\section{LONG-TERM RESULTS OF ENDOSCOPIC BALLOON DILATION IN PATIENTS WITH CROHN'S DISEASE}

The definition of long-term results of EBD differs between studies. In most studies, symptom recurrence is a marker of the "loss of response", and in some cases, the time until the need for surgery/re-intervention is used as a definition. ${ }^{6}$ In all patients who develop a relapse of symptoms, either re-dilation or surgery is the treatment option chosen. Morar et al. defined symptom recurrence as the "loss of response", and observed that after 1,2, or 5 years the rate of re-dilation was approximately $32 \%, 26 \%$, and $2 \%$, respectively. ${ }^{20}$ However, Navaneethan et al. reported in their systematic review that over a median follow-up period of 15-70 months, only $27 \%$ of patients required surgical interventions, and $44 \%$ of 
patients required only one dilation with long-term success. ${ }^{7}$ Notably, median time duration until the requirement of surgery was observed to be 4-33 months ${ }^{7}$ underlining the role of EBD as a bridge to surgery if needed, in a significant proportion of patients. Furthermore, it appears that patients are satisfied with this approach, although surgery may eventually be needed in the long run. We observed that $83 \%$ of patients treated with EBD at our hospital were satisfied with the procedure and indicated that they would again choose this option as first-line treatment although $34 \%$ of these patients eventually underwent surgery during the course of their disease. ${ }^{5}$ Over a median follow-up period of 4.8 years, $55.6 \%$ of patients remained free of stricture-related surgery. ${ }^{5}$

At a time when great progress has been made in all areas of medical therapy, we are still not yet able to predict long-term efficacy of endoscopic stricture therapy. To date, a stricture length $>4 \mathrm{~cm}$ is considered the only significant predictor of recurrence, ${ }^{21}$ and factors, such as inflammatory activity, de novo vs. post-surgical stenosis, passage of the endoscope after dilation or mode of dilation do not appear to influence the long-term outcome. ${ }^{20,21}$ Although Gustavsson et al. have demonstrated smoking to be a risk factor associated with the recurrence of intestinal strictures in patients with $\mathrm{CD}$, our data revealed that smoking habits did not influence the need for surgery after $\mathrm{EBD}^{5,22}$

\section{CONSIDERATIONS IN SPECIAL SITUATIONS IN PATIENTS WITH CROHN'S DISEASE}

Fistulas and stenoses can co-exist in patients with $\mathrm{CD}$ as both entities are commonly a consequence of transmural inflammation. The high-pressure zone associated with stenosis may predispose to fistula formation within or at the proximal aspect of a stenotic area. ${ }^{23}$ We avoid balloon dilation in patients with $\mathrm{CD}$ if fibrostenotic and penetrating diseases are associated with a high grade of inflammation (and prefer institution of medical therapy in such instances). However, we do not consider formation of uncomplicated fistulas as an absolute contraindication to performing balloon dilation. This is important because most fistulas do not heal under a high-pressure challenge even if inflammation is sufficiently suppressed (Fig. 3).

Strictures in the upper gastrointestinal tract or small bowel are also amenable to treatment with EBD. Long-term success has been reported in approximately two-thirds of patients. ${ }^{24}$ Depending on the location, EBD can be performed via a gastroscopy, push enteroscopy, or device-assisted enteroscopy. Accessories used during the EBD procedure need to be adapted to the diameter of the working channel and the length of the endoscope (Fig. 4).

Neoplasms are very rarely associated with a CD stricture; however, they could be fatal if not detected early. There is limited evidence demonstrating that biopsies prior to dilatation increase the subsequent risk of perforation. Therefore, thorough endoscopic evaluation of the stricture is mandatory, if possible, and a low threshold to obtain biopsies is recommended (Fig. 5).

\section{A COMPARISON BETWEEN ENDOSCOPIC BALLOON DILATION AND SURGERY IN PATIENTS WITH CROHN'S DISEASE}

Although EBD is a safe and efficacious procedure, a signif-
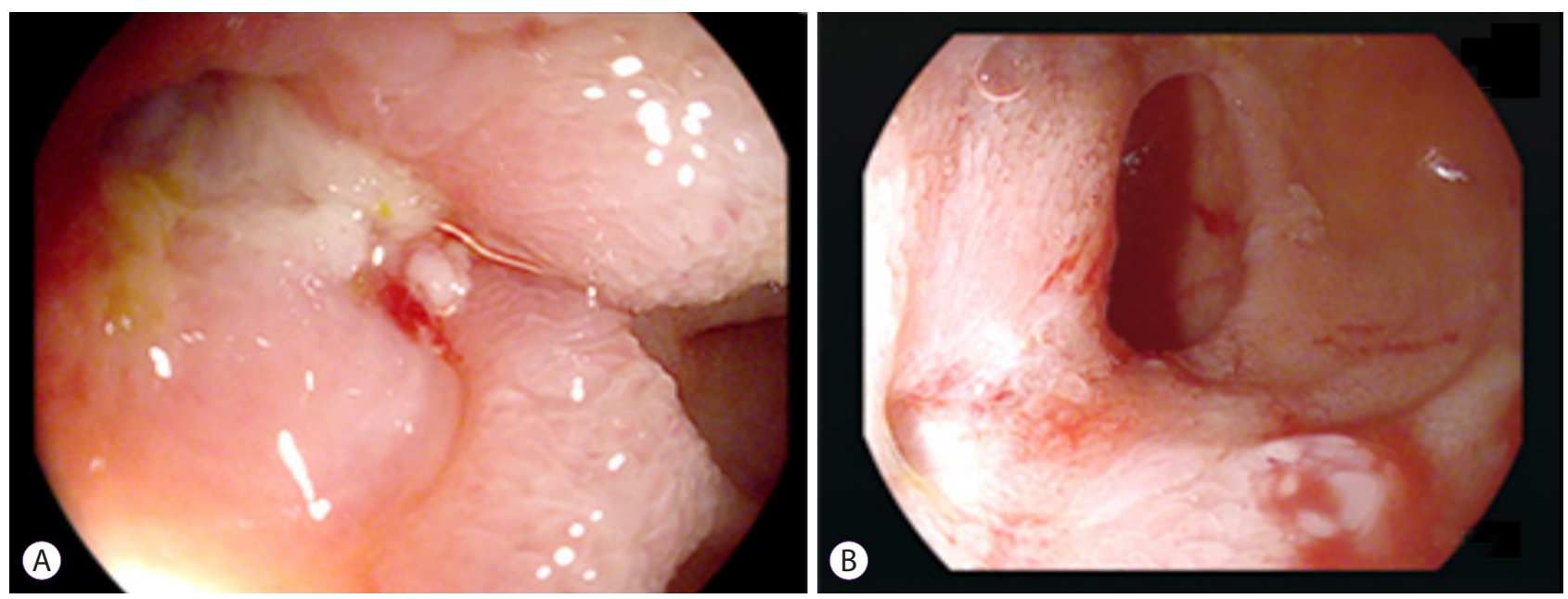

Fig. 3. (A) A suppurative fistula observed at the site of stenosis necessitated medical therapy and balloon dilatation. (B) Four months after dilation, discharge from the fistula is observed to have subsided. 


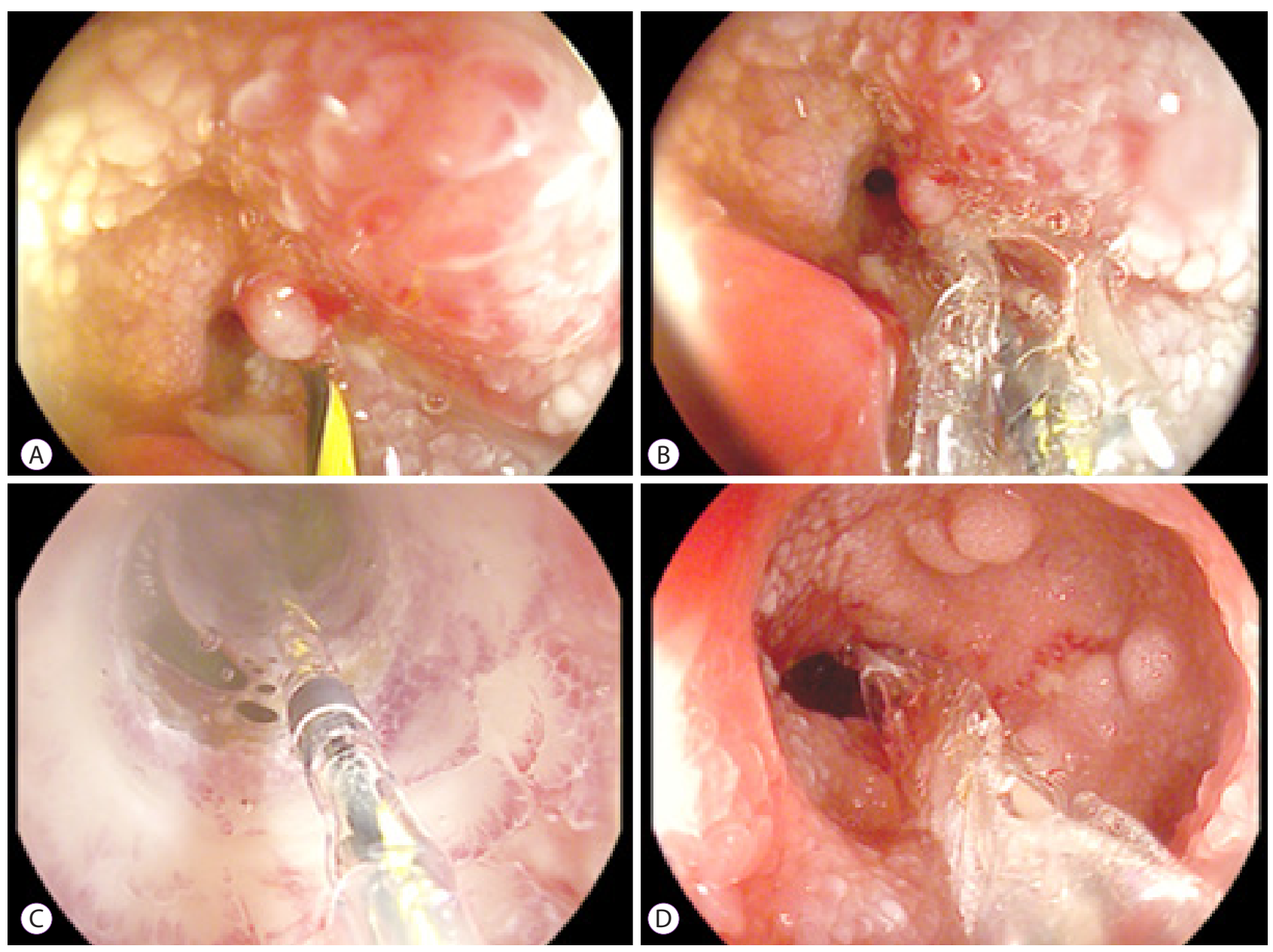

Fig. 4. A site of jejunal stenosis is accessed using single-balloon endoscopy. (A) A guide wire is inserted across the stenosis. (B) A wire-guided balloon is positioned within the stenosis. (C) Water (or contrast)-filled balloons allow direct visual control during dilatation. (D) The stenosis is sufficiently dilated. A second narrowing, which was subsequently dilated is visible distal to the stenosis.

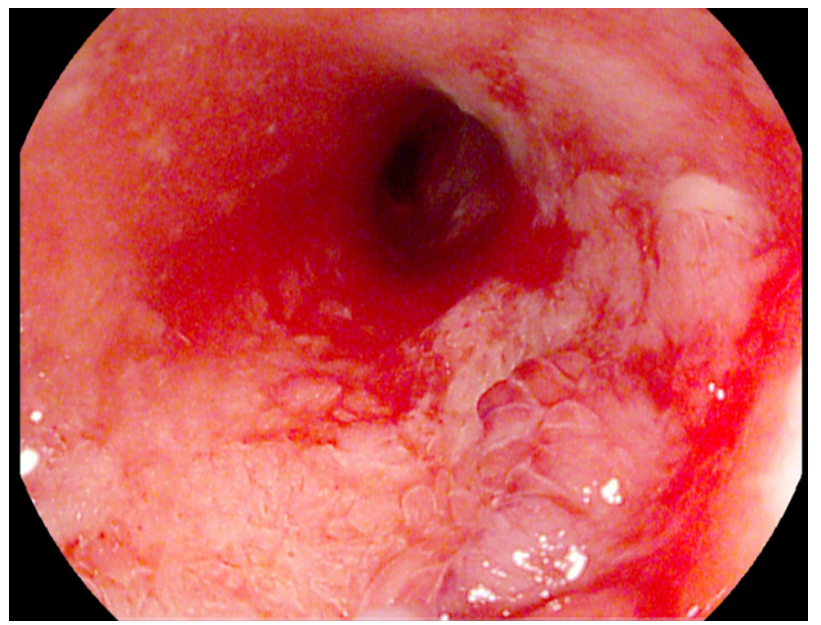

Fig. 5. Histologically confirmed low-grade intestinal neoplasia is observed at the site of a Crohn's disease-associated stenosis (noted at the 4-5 o'clock position).

icant proportion of patients require surgery for a variety of reasons. Depending on the study and patient characteristics, it has been observed that approximately $30 \%-50 \%$ of patients need stricture-related surgery despite having undergone an EBD. ${ }^{3,5,25}$ However, EBD can serve as a bridging treatment modality between medical and surgical therapy because the EBD procedure is easier to perform and potentially lowers the risk of repeat surgery and short bowel syndrome. ${ }^{12}$ Nevertheless, surgery for the management of $\mathrm{CD}$ strictures is also a safe and efficient procedure. Reporting on a retrospective comparison between EBD and surgery, Greener et al. have demonstrated in their cohort study that surgery and EBD show comparable safety profiles (major complications $7.6 \%[\mathrm{EBD}]$ vs. $7.5 \%$ [surgery]). ${ }^{25}$ The need for (re-) surgery or (re-) intervention was significantly lower in the group treated with surgery. ${ }^{25}$ We propose that surgery should be used as a primary intervention in patients presenting with complex strictures $>4 \mathrm{~cm}$, with high-grade inflammation and a high risk of perforation with the potential need for emergency surgery, often resulting in the creation of an ileostomy instead of a direct anastomosis as is commonly performed in cases of elective surgery. ${ }^{12}$ Lian 
et al. have shown that particularly in cases of short ileocolonic anastomotic strictures in patients with $\mathrm{CD}$, the EBD procedure delays the mean time until surgery by approximately 6.5 years. ${ }^{26}$ Therefore, these authors concluded that EBD should be attempted in patients presenting with post-surgical anastomotic strictures with a low risk for persistent active disease although patients at risk for recurrent active disease may benefit from upfront surgical therapy in this situation.

\section{MEDICAL TREATMENT OF CROHN'S DISEASE-RELATED STRICTURES}

The availability of anti-TNF-alpha antibodies, such as infliximab and adalimumab has revolutionized medical therapy of CD. Nevertheless, some reports available in literature describe a higher rate of stricture aggravation because of rapid mucosal healing. ${ }^{3}$ These concerns have not been confirmed in real-life cohorts administered anti-TNF-alpha treatment, and several studies have shown beneficial effects of these agents in the treatment of inflammatory stenosis without an associated increase in the obstruction rates. ${ }^{3} \mathrm{~A}$ large study ( $n=241$ patients) investigating these associations has shown that biologics tend to decrease the need for surgery. ${ }^{27}$ However, inflammatory segments with upstream prestenotic dilatation are less likely to respond to medical therapy. ${ }^{28}$ The role of newer agents, such as the anti-integrin antibody vedolizumab and the interleukin- 23 antibody ustekinumab for the treatment of $\mathrm{CD}$-associated strictures, is yet to be established in real-life cohorts. However, both agents can potentially induce mucosal healing and therefore, are expected to show a positive effect in patients presenting with inflammatory stenoses. To date, no test can effectively quantitate the inflammatory vs. fibrotic component of a stricture, and most strictures show stigmata of both components. It is instructive to know that the inflammatory component of a stricture may be best treated using medical interventions, whereas a fibrostenotic component is not amenable to anti-inflammatory medical treatment. ${ }^{29}$ Presently, targeted antifibrotic treatment is not available for routine treatment in clinical practice.

\section{CONCLUSIONS}

Endoscopic balloon dilatation is safe and shows good short- and long-term results. CD-associated strictures require an interdisciplinary treatment approach. Medical, endoscopic, and surgical approaches are complementary and can be combined, if and as needed, or used sequentially to optimize patient satisfaction and treatment success.

Conflicts of Interest

All authors declare that we received fees and/or travel support from AbbVie, Merck Sharp \& Dohme, Janssen Pharma, Falk Foundation, Novartis, and Takeda.

\section{REFERENCES}

1. Wehkamp J, Götz M, Herrlinger K, Steurer W, Stange EF. Inflammatory bowel disease. Dtsch Arztebl Int 2016;113:72-82.

2. Cosnes J, Cattan S, Blain A, et al. Long-term evolution of disease behavior of Crohn's disease. Inflamm Bowel Dis 2002;8:244-250.

3. Bharadwaj S, Fleshner P, Shen B. Therapeutic armamentarium for stricturing Crohn's disease: medical versus endoscopic versus surgical approaches. Inflamm Bowel Dis 2015;21:2194-2213.

4. Rutgeerts P, Geboes K, Vantrappen G, Beyls J, Kerremans R, Hiele M. Predictability of the postoperative course of Crohn's disease. Gastroenterology 1990;99:956-963.

5. Rueda Guzmán A, Wehkamp J, Kirschniak A, Naumann A, Malek NP, Goetz M. Endoscopic balloon dilatation of Crohn's-associated intestinal strictures: high patient satisfaction and long-term efficacy. United European Gastroenterol J 2016;4:794-799.

6. Hirai F. Current status of endoscopic balloon dilation for Crohn's disease. Intest Res 2017;15:166-173.

7. Navaneethan U, Lourdusamy V, Njei B, Shen B. Endoscopic balloon dilation in the management of strictures in Crohn's disease: a systematic review and meta-analysis of non-randomized trials. Surg Endosc 2016;30:5434-5443.

8. Mueller T, Rieder B, Bechtner G, Pfeiffer A. The response of Crohn's strictures to endoscopic balloon dilation. Aliment Pharmacol Ther 2010;31:634-639.

9. Van Assche G, Vermeire S, Rutgeerts P. Endoscopic therapy of strictures in Crohn's disease. Inflamm Bowel Dis 2007;13:356-358; discussion 362363.

10. Koltun WA. Dangers associated with endoscopic management of strictures in IBD. Inflamm Bowel Dis 2007;13:359-361; discussion 362-363.

11. Bettenworth D, Lopez R, Hindryckx P, Levesque BG, Rieder F. Heterogeneity in endoscopic treatment of Crohn's disease-associated strictures: an international inflammatory bowel disease specialist survey. J Gastroenterol 2016;51:939-948.

12. Chen M, Shen B. Endoscopic therapy in Crohn's disease: principle, preparation, and technique. Inflamm Bowel Dis 2015;21:2222-2240.

13. Rieder F, Latella G, Magro F, et al. European Crohn's and colitis organisation topical review on prediction, diagnosis and management of fibrostenosing Crohn's disease. J Crohns Colitis 2016;10:873-885.

14. Dillman JR, Smith EA, Sanchez R, et al. Prospective cohort study of ultrasound-ultrasound and ultrasound-MR enterography agreement in the evaluation of pediatric small bowel Crohn disease. Pediatr Radiol 2016;46:490-497.

15. Ripollés T, Rausell N, Paredes JM, Grau E, Martínez MJ, Vizuete J. Effectiveness of contrast-enhanced ultrasound for characterisation of intestinal inflammation in Crohn's disease: a comparison with surgical histopathology analysis. J Crohns Colitis 2013;7:120-128.

16. ASGE Standards of Practice Committee, Anderson MA, Ben-Menachem T, et al. Management of antithrombotic agents for endoscopic procedures. Gastrointest Endosc 2009;70:1060-1070.

17. Veitch AM. Endoscopy in patients on antiplatelet agents and anticoagulants. Curr Treat Options Gastroenterol 2017;15:256-267.

18. Gustavsson A, Magnuson A, Blomberg B, Andersson M, Halfvarson J, Tysk C. Endoscopic dilation is an efficacious and safe treatment of intestinal strictures in Crohn's disease. Aliment Pharmacol Ther 2012;36:151158. 
19. Hirai F, Matsui T, Yao K, Sou S, Seki T. Efficacy of carbon dioxide insufflation in endoscopic balloon dilation therapy by using double balloon endoscopy. Gastrointest Endosc 2007;66(3 Suppl):S26-S29.

20. Morar PS, Faiz O, Warusavitarne J, et al. Systematic review with meta-analysis: endoscopic balloon dilatation for Crohn's disease strictures. Aliment Pharmacol Ther 2015;42:1137-1148.

21. Hassan C, Zullo A, De Francesco V, et al. Systematic review: endoscopic dilatation in Crohn's disease. Aliment Pharmacol Ther 2007;26:14571464.

22. Gustavsson A, Magnuson A, Blomberg B, Andersson M, Halfvarson J, Tysk C. Smoking is a risk factor for recurrence of intestinal stricture after endoscopic dilation in Crohn's disease. Aliment Pharmacol Ther 2013;37:430-437.

23. Oberhuber G, Stangl PC, Vogelsang H, Schober E, Herbst F, Gasche C. Significant association of strictures and internal fistula formation in Crohn's disease. Virchows Arch 2000;437:293-297.

24. Fukumoto A, Tanaka S, Yamamoto H, et al. Diagnosis and treatment of small-bowel stricture by double balloon endoscopy. Gastrointest Endosc 2007;66(3 Suppl):S108-S112.
25. Greener T, Shapiro R, Klang E, et al. Clinical outcomes of surgery versus endoscopic balloon dilation for stricturing Crohn's disease. Dis Colon Rectum 2015;58:1151-1157.

26. Lian L, Stocchi L, Remzi FH, Shen B. Comparison of endoscopic dilation vs surgery for anastomotic stricture in patients with Crohn's disease following ileocolonic resection. Clin Gastroenterol Hepatol 2017;15:1226-1231.

27. Nepal S, Bahuva R, Shen B, Atreja A. Early initiation of biologics and immunomodulators decrease the need for surgery in enterography proven stricturing Crohn's disease. Gastroenterology 2012;142(5 Suppl 1):S64-S65 .

28. Lawrance IC, Welman CJ, Shipman P, Murray K. Correlation of MRI-determined small bowel Crohn's disease categories with medical response and surgical pathology. World J Gastroenterol 2009;15:33673375.

29. Bouguen G, Trouilloud I, Siproudhis L, et al. Long-term outcome of non-fistulizing (ulcers, stricture) perianal Crohn's disease in patients treated with infliximab. Aliment Pharmacol Ther 2009;30:749-756. 